\title{
The Effect of Hypotension on Brain Energy State during Prolonged Neonatal Seizure
}

\author{
BARRETT E. COWAN, RICHARD S. K. YOUNG, RICHARD W. BRIGGS, DANIEL LU, AND \\ MARIA SENA \\ Departments of Pediatrics and Neurology, Stanford University, Stanford, [B.E.C., D.L., M.S.] and Yale \\ University, New Haven, Connecticut [R.S.K.Y.]; and Departments of Radiology and Biological Chemistry, The \\ Pennsylvania State University, Hershey, Pennsylvania [R.W.B.]
}

\begin{abstract}
Prolonged seizures in the human neonate may be complicated by systemic hypotension. To examine the effect of systemic hypotension on brain metabolic state during seizure, neonatal dogs were made hypotensive (by exsanguination) during bicuculline-induced seizure. Measurement of regional cerebral blood flow showed that moderate hypotension did not impair cerebral perfusion during seizure. Measurement of brain energy state with in vivo ${ }^{31} \mathrm{P}$ nuclear magnetic resonance spectroscopy disclosed a similar pattern of alteration of high energy phosphates in animals subjected to seizure or to the combination of seizure and hypotension. The additional metabolic stress imposed by moderate hypotension during seizure in the neonatal dog appears to be slight. (Pediatr Res 21:357$361,1987)$
\end{abstract}

\section{Abbreviations}

CBF, cerebral blood flow

NMR, nuclear magnetic resonance

$\mathrm{PCr}$, phosphocreatine

ATP, adenosine triphosphate

$\mathbf{P}_{\mathrm{i}}$, inorganic phosphate

$\mathbf{p H}_{\text {, }}$, intracellular pH

Clinical and experimental evidence show that systemic hypotension may occur during the course of prolonged seizure (1-3). Nonetheless, the physiologic and metabolic consequences of hypotension on the outcome of neonatal seizure is unclear (4). Because metabolic demands on the brain increase greatly during seizure, it is hypothesized that even a modest reduction in $\mathrm{CBF}$ produces relative ischemia and further tissue damage.

Autoregulation of $\mathrm{CBF}$ ordinarily maintains cerebral perfusion during systemic hypotension $(5,6)$, but may be impaired during seizure $(4,7)$. However, use of the term, autoregulation, may be inappropriate since the response of the cerebral circulation to seizure is not a simple maintenance of blood flow, but rather a sustained increase of $150-200 \%(8,9)$. The goal of this study was to determine whether the expected compensatory increase in $\mathrm{CBF}$ which occurs during seizure is affected by moderate hypotension and whether there is subsequent compromise of brain energy state.

Received September 16, 1986; accepted November 17, 1986.

Correspondence and reprint requests to Richard S. K. Young, M.D., Department of Pediatrics, Yale University School of Medicine, 333 Cedar Street, New Haven, CT 06510.

Supported by NIH Grants R01-24605 (RSKY) and R23-CA39627 (RWB). R.S.K.Y. is a recipient of a Clinician-Scientist Award from the American Heart Association

\section{METHODS}

The method has been previously described in detail $(8,9)$. Briefly, mongrel dogs (1-10 days old) were anesthetized for 5 min with halothane while undergoing tracheostomy. Pancuronium bromide was administered $(0.3 \mathrm{ml}$ intraperitoneal) and halothane discontinued. Animals were mechanically ventilated with $30 \% \mathrm{O}_{2}$ and minute ventilation was adjusted to afford normoxia $\left(\mathrm{p}_{\mathrm{a}} \mathrm{O}_{2}, 80-120 \mathrm{~mm} \mathrm{Hg}\right)$ and normocarbia $\left(\mathrm{pCO}_{2}, 30\right.$ $40 \mathrm{~mm} \mathrm{Hg}$ ). Seventy percent nitrous oxide was used for analgesia and all sites of incision were infiltrated with local anesthetic (xylocaine, 1\%). The femoral artery and vein were cannulated to permit monitoring of blood pressure, withdrawal of blood samples, and infusion of drugs $(8,9)$.

Animals were randomized to one of four study groups: control, hypotension, seizure, and seizure-hypotension. Animals in the seizure group received bicuculline (Sigma, $2 \mathrm{mg} / \mathrm{kg}$ intravenous), an alkaloid which blocks the inhibitory neurotransmitter, $\gamma$ amino-butyric acid. Animals in the control group were given an equivalent volume of $0.9 \%$ saline. Animals in the hypotension groups were exsanguinated until MABP had been reduced by one-half (from approximately 78 to $40 \mathrm{~mm} \mathrm{Hg}$ ). A target of 40 $\mathrm{mm} \mathrm{Hg}$ was selected because prior studies have shown that perfusion in certain brain regions may begin to fail below this level (6). Animals in the seizure-hypotension group were rendered hypotensive but were also administered bicuculline.

EEG was continuously monitored with scalp electrodes and a Grass polygraphic recorder. Autoradiographic measurement of $\mathrm{CBF}$ with $\left[{ }^{14} \mathrm{C}\right]$ iodoantipyrine $(6,8,10)$ was performed in a parallel group of animals at the end of a $45-\mathrm{min}$ period of experimental observation.

${ }^{31} \mathrm{P}$ NMR data were collected utilizing a 1.89 Tesla, $26-\mathrm{cm}$ bore diameter superconducting magnet (Oxford Instruments), a Nicolet 1280 computer, and a transmitter-receiver coil tuned for phosphorus (32.5 MHz partially flattened Helmholtz coil encompassing approximately $3.5 \times 4 \times 1.5 \mathrm{~cm}$ of both gray and white matter). Reflection of cranial muscle was not attempted because postmortem examination revealed little muscle tissue over the skull. Moreover, previous experiments $(8,9)$ disclosed negligible signal obtained from this source. The underlying broad spectral component due to cranial bone and phospholipids was partially removed by frequency selective saturation in the region between the $\alpha$ - and $\beta$-phosphate ATP peaks $(11,12)$ using a "DANTE (13)" pulse sequence (1024 eight $\mu \mathrm{s}$ pulses separated by $250-\mu \mathrm{s}$ intervals and attenuated by $12 \mathrm{~dB})$. A pulse width of $36 \mu \mathrm{s}$ provided optimal signal to noise (approximately 12.5:1 for ATP after 300 one-s scans). Rapid pulsing was performed to increase sensitivity per unit time (14). Optimization of the homogeneity of the magnet was performed by observing the proton signal of water.

Baseline control spectra were collected for $15 \mathrm{~min}$. Following 

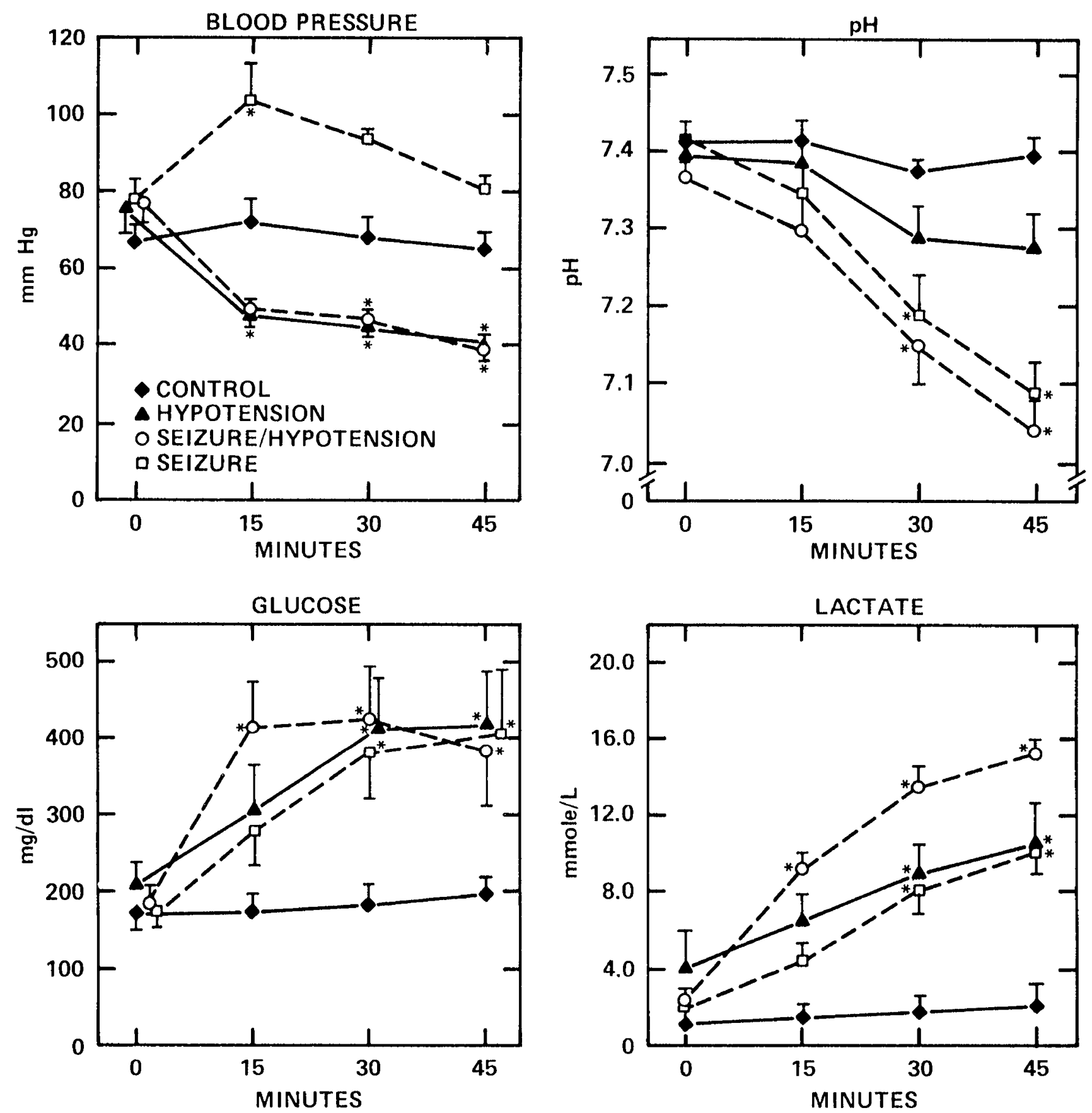

$M E A N \pm S E$

${ }^{*} \mathrm{p}<0.05$ vs $0 \mathrm{~min}$

Fig. 1. Systemic changes. Animals in the hypotension and seizure-hypotension groups are significantly hypotensive. Hyperglycemia occurs in all three experimental groups by $30 \mathrm{~min}$. Acidosis is most prominent in the seizure and seizure-hypotension groups. 
A. BEFORE HYPOTENSION

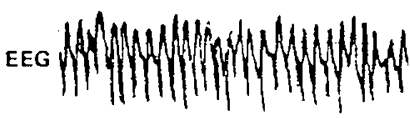

Mean Blood Pressure $=68 \mathrm{~mm} \mathrm{Hg}$
B. HYPOTENSION

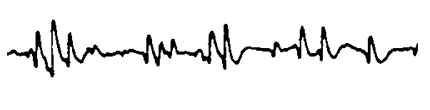

Mean Blood Pressure $=\mathbf{4 0} \mathrm{mm} \mathrm{Hg}$
DOG 518

Fig. 2. EEG in bicuculline injected neonatal dog before and during hypotension. Spike and slow wave discharge is present both before and during hypotension in bicuculline injected neonatal dog. However, discharges during hypotension are less regular.

this, animals in the seizure group or seizure-hypotension group were administered bicuculline and animals in the hypotension or seizure-hypotension group were made hypotensive. Three sets of spectra were then acquired $(0-15,16-30$, and $31-45 \mathrm{~min})$. The Nicolet 1280 computer program ("NMRCAP") was utilized to determine areas of individual resonances in spectral regions where peak overlap occurred. Brain $\mathrm{pH}_{\mathrm{i}}$ was determined by noting the chemical shift of the $P_{i}$ peak with respect to that of $\mathrm{PCr}$, which is constant over the physiological range of $\mathrm{pH}$. Literature values (15) of cerebral intracellular concentrations of ions and metabolites were used to prepare a standard $\mathrm{pH} / \mathrm{p}_{\mathrm{i}}$ titration curve (8).

Immediately after collection of NMR spectra, brains of animals were frozen in situ with liquid nitrogen (16) and stored at $-80^{\circ} \mathrm{C}$. They were later dissected under liquid nitrogen irrigation, extracted $\left(\mathrm{HClO}_{4}\right)$, and spectrophotometrically assayed for concentrations of glucose, lactate, ATP, and $\mathrm{PCr}$ according to standard enzymatic techniques (17).

Analysis of variance and the Newman-Keuls post hoc test were used to determine statistically significant $(p<0.05)$ intergroup and intragroup differences, respectively. Twenty-five animals were used in the CBF studies (Table 1); 37 animals were used in the metabolism studies (control nine; hypotension six; seizure 10 , seizure-hypotension 12 ). Values from six of the 10 seizure animals have been previously reported (9).

\section{RESULTS}

Arterial blood pressure was transiently increased in animals in the seizure group (Fig. 1). In contrast, blood pressure was significantly lowered in both the hypotension and the seizure-hypotension groups. Serum glucose and lactate levels increased in all three experimental groups. Arterial $\mathrm{pH}$ decreased in both seizure and seizure-hypotension animals but was unchanged in the control and hypotension animals.
An EEG pattern of low voltage fast activity was present in both control animals and animals subjected to hypotension alone. Continuous high voltage spike or spike and slow wave discharges were observed in both the seizure and seizure-hypotension groups, although the discharges were less frequent and more irregular in the latter group (Fig. 2).

CBF was not significantly reduced in animals subjected to hypotension alone as compared to controls. However, increases in CBF occurred in both the seizure and the seizure-hypotension groups. There were no significant differences in CBF between seizure and seizure-hypotension groups (Table 1).

${ }^{31} \mathrm{P}$ NMR measurements showed no significant changes in brain metabolic state in animals subjected only to hypotension (Fig. 3). In contrast, there were parallel reductions of $\mathrm{PCr}, \mathrm{PCr} /$ $\mathrm{P}_{\mathrm{i}}$, and $\mathrm{pH}_{\mathrm{i}}$ and a concomitant increase in $\mathrm{P}_{\mathrm{i}}$ in both the seizure and the seizure-hypotension groups. The ATP level and the ATP/ $P_{i}$ ratio were significantly decreased in the seizure-hypotension group compared to the control group. At no time were there significant differences in the ATP or $\mathrm{PCr}$ levels or $\mathrm{PCr} / \mathrm{P}_{\mathrm{i}}$ or $A T P / P_{i}$ ratios between the seizure-hypotension and the seizure animals.

In vitro assay of brain metabolites disclosed a significant increase in lactate concentrations in both the seizure and the seizure-hypotension groups compared to that of control animals. $\mathrm{PCr}$, ATP, and glucose levels were significantly reduced in the seizure-hypotension group as compared to either the control or hypotension group (Fig. 4).

\section{DISCUSSION}

The metabolic requirements of the brain increase considerably during seizure. It has therefore been hypothesized that if hypotension occurs during seizure, cerebral ischemia may ensue and produce brain injury (18). The mechanisms controlling CBF during seizure in the neonatal dog are uncertain. Nonetheless, previous experiments have shown that CBF remains at high levels long after the initial systemic hypertension has passed (8). The present data extend those findings by showing that both $\mathrm{CBF}$ and paroxysmal EEG activity continue unabated during the combination of hypotension and seizure.

Adult animals and developmentally more precocious neonates may not be as tolerant of hypotension during seizure. While mild hypotension during seizure in the adult experimental animal does not increase tissue injury (19), moderate hypotension during seizure in the adult rat leads to cerebral ischemia and an isoelectric EEG (20). A preliminary report suggests that the neonatal pig is also incapable of augmenting CBF during the combination of seizure and hypotension (21). The neonatal dog may be more tolerant of hypotension during seizure because of lower baseline blood pressure $(5,6)$.

Changes in brain metabolic parameters were approximately parallel in the seizure-hypotension and seizure groups. Brain lactate measured in vitro and levels of $\mathrm{PCr}$ and $\mathrm{P}_{\mathrm{i}}$ and the $\mathrm{PCr}$ /

Table 1. $C B F(\text { mean } \pm S E, m l / 100 \mathrm{~g} / \mathrm{min})^{*}$

\begin{tabular}{|c|c|c|c|c|}
\hline Brain region & Control & Hypotension & Seizure-hypotension & Seizure \\
\hline Frontal cortex & $40 \pm 3$ & $35 \pm 3$ & $62 \pm 13 \dagger \ddagger$ & $65 \pm 4 \uparrow \ddagger$ \\
\hline Parietal cortex & $39 \pm 2$ & $33 \pm 2$ & $62 \pm 12 \dagger \ddagger$ & $67 \pm 5 \dagger \ddagger$ \\
\hline Caudate & $38 \pm 4$ & $24 \pm 3$ & $55 \pm 12 \ddagger$ & $69 \pm 8 \dagger \ddagger$ \\
\hline Hippocampus & $35 \pm 3$ & $30 \pm 4$ & $57 \pm 5 \dagger+$ & $65 \pm 5 \dagger \ddagger$ \\
\hline Thalamus & $52 \pm 4$ & $49 \pm 4$ & $97 \pm 27 \dagger+$ & $97 \pm 8 \dagger \ddagger$ \\
\hline Inf. colliculus & $50 \pm 7$ & $50 \pm 9$ & $92 \pm 5 \dagger \neq$ & $82 \pm 6$ \\
\hline Medulla & $53 \pm 5$ & $57 \pm 6$ & $96 \pm 41+\div$ & $91 \pm 6 \dagger \neq$ \\
\hline Spinal cord & $51 \pm 6$ & $62 \pm 13$ & $87 \pm 6 \dagger$ & $75 \pm 5$ \\
\hline White matter & $22 \pm 2$ & $17 \pm 1$ & $46 \pm 17+$ & $36 \pm 4$ \\
\hline
\end{tabular}

${ }^{*}$ Number of animals: control 12; hypotension 3; seizure-hypotension 3; seizure 7. No significant differences exist between seizure-hypotension and seizure animals.

$\dagger p<0.05$ versus control.

$\ddagger p<0.05$ versus hypotension. 

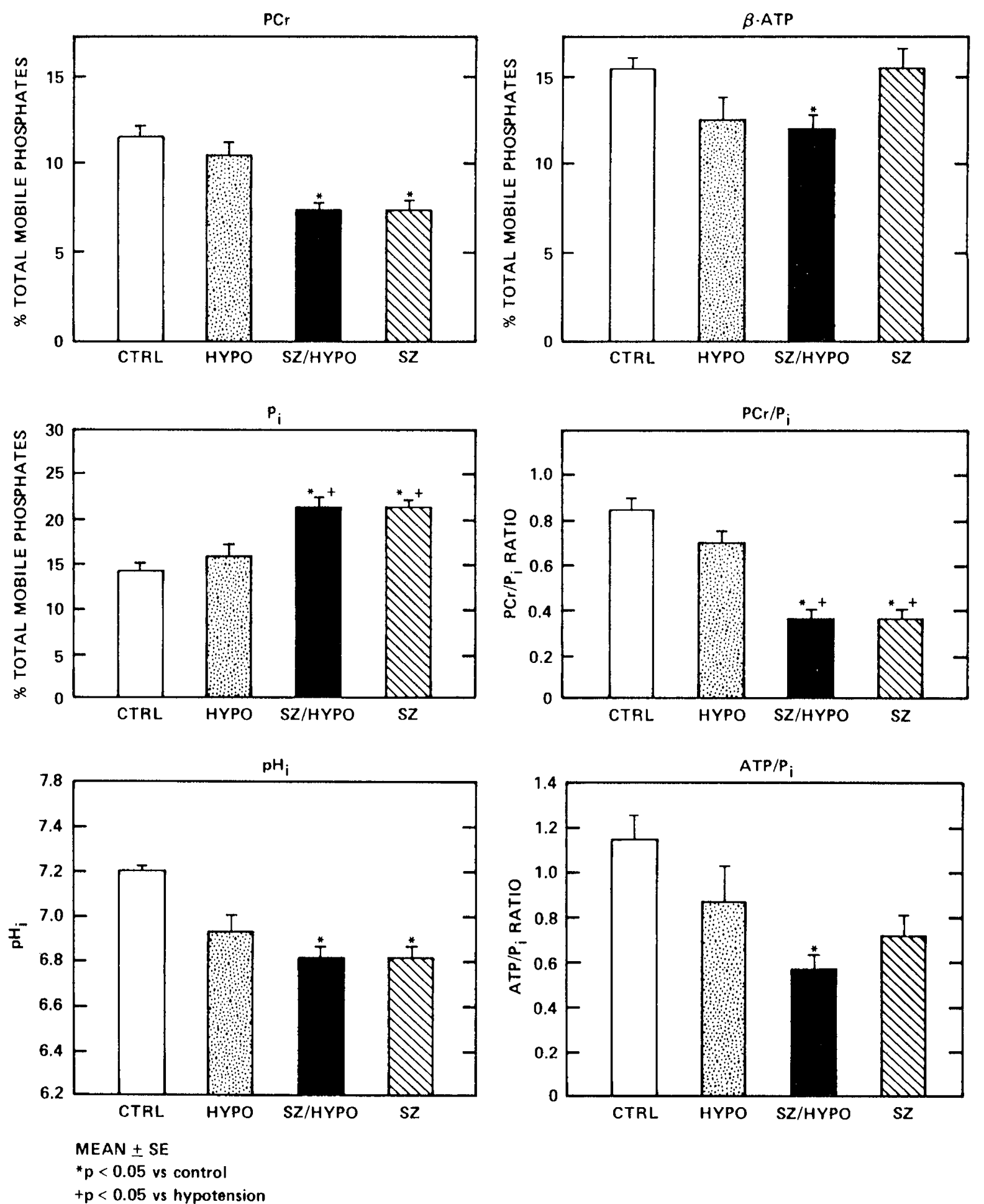

Fig. 3. Brain high energy phosphate metabolism. After 45 min there are parallel changes in phosphocreatine, $\mathrm{P}_{\mathrm{i}}, \mathrm{PCr} / \mathrm{P}_{\mathrm{i}}$ ratio, and intracellular $\mathrm{pH}$ in the seizure and seizure-hypotension groups. $\beta$ - $\mathrm{ATP}$ and $\mathrm{ATP} / \mathrm{P}_{\mathrm{i}}$ ratio are significantly reduced only in the seizure-hypotension group. $C T R L$, control; $H Y P O$, hypotension; $S Z / H Y P O$, seizure-hypotension; $S Z$, seizure. 

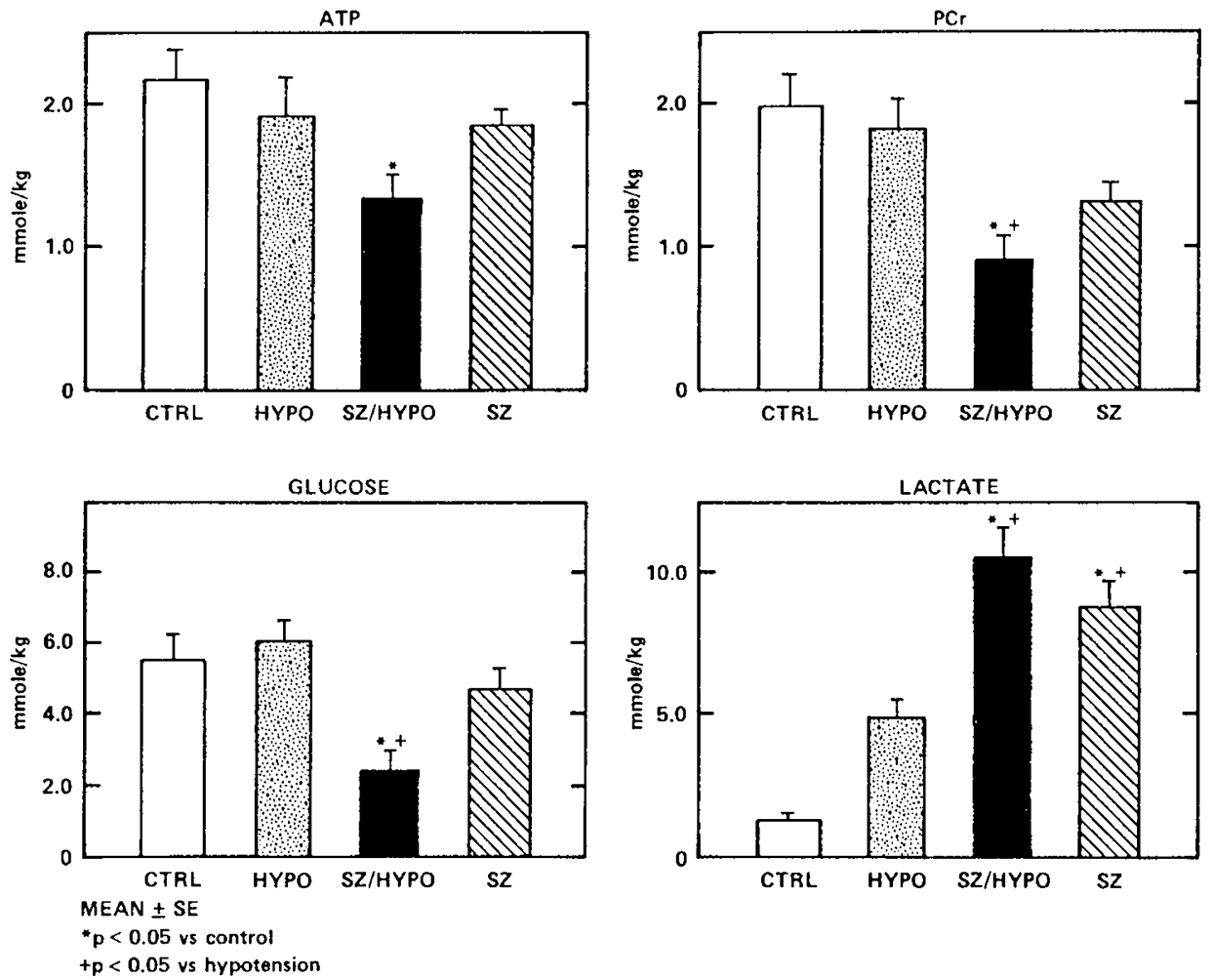

Fig. 4. In vitro brain metabolites. Brain lactate is elevated in both seizure and seizure-hypotension groups. PCr, ATP, and glucose are reduced significantly compared to controls only in the seizure-hypotension group. There are no statistically significant differences between values in the seizure and the seizure-hypotension groups.

$P_{i}$ ratio measured in vivo were reduced to a similar degree in both of these groups compared to the control group. ATP and the $\mathrm{ATP} / \mathrm{P}_{\mathrm{i}}$ ratio were slightly decreased only in the seizurehypotension group. While it could be postulated that the seizurehypotension group fared worse than the seizure group, it should be noted that at no point were the differences between seizure and seizure-hypotension animals statistically significant.

In summary, cerebral perfusion during seizures in the neonatal dog remained at increased levels during a period of moderate hypotension. Consequently, the additional metabolic derangement induced by systemic hypotension during seizure in this neonatal animal was slight. Metabolic stress induced by the increased cerebral activity during seizure may be a primary effect not substantially exacerbated by concurrent moderate hypotension.

\section{REFERENCES}

1. Glaser GH 1983 Medical complications of status epilepticus. Adv Neurol 34:395-398

2. Prichard JW, Alger JR, Behar KL, Petroff OAC, Shulman RG 1983 Cerebral metabolic studies in vivo by ${ }^{31}$ P NMR. Proc Natl Acad Sci USA 80:27482751

3. Young RSK, Fripp RR, Yagel SK, Werner JC, McGrath G, Schuler HG 1985 Cardiac dysfunction during status epilepticus in the neonatal pig. Ann Neurol 18:291-297

4. Volpe JJ 1981 Neonatal seizures. In: Volpe JJ (ed) Neurology of the Newborn. WB Saunders, Philadelphia, p 128

5. Hernandez MJ, Brennan RW, Bowman GS 1980 Autoregulation of CBF in the newborn dog. Brain Res 184:199-202

6. Young RSK, Hernandez MJ, Yagel SK 1982 Selective reduction of blood flow to white matter during hypotension in newborn dogs: a possible mechanism of periventricular leukomalacia. Ann Neurol 12:445-448

7. Astrup J, Blennow G, Nilsson B 1979 Effects of reduced cerebral blood flow upon EEG pattern, cerebral extracellular potassium, and energy metabolism in the rat cortex during bicuculline-induced seizures. Brain Res 177:115126
8. Young RSK, Osbakken MD, Briggs RW, Yagel SK, Rice DW, Goldberg S $1985{ }^{31} \mathrm{P}$ NMR study of cerebral metabolism during prolonged seizures in the neonatal dog. Ann Neurol 18:14-20

9. Young RSK, Briggs RW, Yagel SK, Gorman I $1986{ }^{31} \mathrm{P}$ Nuclear magnetic resonance study of the effect of hypoxemia on neonatal status epilepticus. Pediatr Res 20:581-586

10. Sakurada O, Kennedy C, Jehle J, Brown JD, Carbin GL, Sokoloff L 1978 Measurement of local cerebral blood flow with iodo $\left({ }^{14} \mathrm{C}\right)$ antipyrine. Am J Physiol 234:H59-H66

11. Ackerman JJH, Evelhoch JL, Berkowitz BH, Kichura GM, Deuel RK, Lown KS 1984 Selective suppression of the cranial bone resonance from ${ }^{31} \mathrm{P}$ NMR experiments with rat brain in vivo. J Magn Reson 56:318-322

12. Gonzalez-Mendez R, Litt L, Koretsky AP, Von Colditz J, Weiner MW, James TL 1984 Comparison of ${ }^{\text {s1 }} \mathrm{P}$ NMR spectra of in vivo rat brain using convolution difference and saturation with a surface coil. Source of the broad component in the brain spectrum. J Magn Reson 57:526-533

13. Morris GA, Freeman R 1978 Selective excitation in Fourier transform nuclear magnetic resonance. J Magn Reson 29:433-462

14. Evelhoch JL, Crowley MG, Ackerman JJH 1984 Signal to noise optimization and observed volume localization with circular surface coils. J Magn Reson 56:110-124

15. Veloso D, Guynn RW, Oskarsson M, Veech RL 1973 The concentrations of free and bound magnesium in rat tissues: relative constancy of frec $\mathrm{mg}^{++}$ concentrations. J Biol Chem 248:481 1-4819

16. Ratcheson RA 1980 Funnel freezing for preservation of intermediary metabolites in rats. In: Passonneau JV, Hawkins RA, Lust WD, Welsh FA (eds) Cerebral Metabolism and Neural Function. Baltimore, Williams \& Wilkins, pp 20-28

17. Bergmeyer HV 1974 Methods of Enzymatic Analysis. New York, Academic Press

18. Meldrum BS, Nilsson B 1976 Cerebral blood flow and metabolic rate early and late in prolonged epileptic seizures induced in rats by bicuculline. Brain 99:523-542

19. Soderfeldt B, Kalimo H, Olsson Y, Siesjo BK 1983 Influence of systemic factors on experimental epileptic brain injury. Acta Neuropathol 60:81-91

20. Blennow G, Nilsson B, Siesjo BK 1985 Influence of reduced oxygen availability on cerebral metabolic changes during bicuculline-induced seizures in rats. J Cereb Blood Flow Metab 5:439-445

21. Hascoet JM, Monin P, Vert P 1986 Loss of cerebral blood flow autoregulation in the postictal period. Pediatr Res 20:350A 